\title{
Ergebnisse des SABCS 2007: Was ist für die Klinik interessant?
}

\author{
Gesprächsleitung: J. Wolfgang Janni und Ingo Bauerfeind, München \\ Teilnehmer: Tanja Fehm, Tübingen Bernd Gerber, Rostock Jens Huober, St. Gallen \\ Roland Reitsamer, Salzburg Andreas Schneeweiss, Heidelberg Christoph Thomssen, Halle/Saale
}

\section{Frage 1: Gibt es in Ergänzung zu den konventionellen prognostischen und prädiktiven Faktoren derzeit ein Muss für die Bestimmung zusätzlicher immunhistochemischer oder molekularer Marker?}

Fehm: Derzeit gibt es keinen Marker, der auf Grund der Datenlage in das Routine-Panel bestehend aus ER, PR und HER2 aufgenommen werden muss.

Gerber: Derzeit ergibt sich aus meiner Sicht keine Änderung in der Bestimmung von prognostischen/prädiktiven Faktoren oder molekularer Marker. Prognoseparameter haben wir mehr als ausreichend! Nur für eine begrenzte Anzahl von Substanzen (Antihormontherapie, Trastuzumab) ist derzeit die Definition des Targets erforderlich. Andere Parameter wie z.B. Topo-II $\alpha$ für das Ansprechen auf Anthrazykline oder CYPIId6 für das Ansprechen auf Tamoxifen sind derzeit nicht durch prospektiv randomisierte Studien abgesichert und sollten deshalb auch nicht bestimmt werden. Gleiches gilt auch für die Bestimmung von Gensignaturen in der adjuvanten Therapie. Solange keine Ergebnisse aus den laufenden Studien (MINDACT-, TAILORx-Trial) vorliegen, kann eine routinemäßige Anwendung nicht empfohlen werden.

Huober: Nein. Neben den konventionellen Faktoren wie Tumorgröße und Lymphknotenstatus und dem Hormonrezeptorstatus, der eher ein prädiktiver als ein prognostischer Faktor ist und dem HER2-Status, der hauptsächlich als prädiktiver Faktor genutzt wird, gibt es momentan keine weiteren Faktoren, die ein Muss sind. PAI-1 und uPA sind gut validierte Faktoren und können genutzt werden. Sie werden gerade in der NNBC-3-Studie bei nodal negativen Patientinnen untersucht. Gensignaturen wie der Amsterdam Score und auch der Onkotype-DX-Test sind noch nicht genügend validiert und sollten nicht in der klinischen Routine eingesetzt werden. Die beiden Studien, die diese Tests untersuchen, sollten abgewartet werden.

Reitsamer: Gen-Expressions-Signaturen werden zunehmend eine Rolle für das Ansprechen einer bestimmten Therapie (Chemotherapie-Response) und für die Prognose eines guten oder schlechten Outcome spielen. «Low-risk-» und «Highrisk-Genprofile» lassen sich sicher unterscheiden, die Datenlage ist sehr vielversprechend, aber es ist noch zu früh für den routinemäßigen Einsatz. Validierungsstudien müssen noch abgewartet werden. Die Marker uPA/PAI-1 sind an sich gut etabliert, allerdings werden sie selten routinemäßig eingesetzt.

Schneewei $\beta$ : Nein, im klinischen Alltag sollten keine weiteren Faktoren für Indikationsstellung und Therapieauswahl benutzt werden. Moderne molekulare Marker wie die GenExpressions-Signaturen (z.B. Recurrence score, AmsterdamSignatur) haben bisher nur in retrospektiven, Hypothesengenerierenden Studien ihre Überlegenheit gegenüber den konventionellen Faktoren gezeigt. Diese Ergebnisse müssen vor dem breiten klinischen Einsatz erst durch die laufenden prospektiven Studien (TAILORx, MINDACT) bestätigt werden.

Thomssen: Obligate molekulare Marker mit prognostischer und/oder prädiktiver Bedeutung sind Östrogenrezeptorstatus, Progesteronrezeptorstatus und die HER2-Expression. In vielen Leitlinien wie denen der Arbeitsgemeinschaft Gynäkologische Onkologie (www.ago-online.de) oder den ASCO Recommendations for the Use of Tumor Markers [1] wird daneben die Bestimmung der Invasionsfaktoren uPA und PAI-1 empfohlen. Die Genexpressionsanalyse mittels Microarrays oder andere Multiparameter-Tests werden dagegen derzeit in Deutschland nicht als reif für die Nutzung außerhalb von Studien gesehen.

\begin{tabular}{ll}
\hline KARGER & @ 2008 S. Karger GmbH, Freiburg \\
Fax +497614520714 & Accessible online at: \\
$\begin{array}{l}\text { E-mail Information@Karger.de } \\
\text { www.karger.com }\end{array}$ & www.karger.com/brc
\end{tabular}

\section{KARGER}

www.karger.com 
Frage 2: Wird die Hochdosischemotherapie beim Mammakarzinom in bestimmten Subkollektiven eine Renaissance erfahren?

Fehm: Zum Thema Hochdosischemotherapie beim Mammakarzinom wurde eine Metaanalyse von Berry et al. vorgestellt. Insgesamt gingen die Ergebnisse von 15 randomisierten Studien ( $n=6210$ Patientinnen) in die Auswertung ein, die Hochdosis- und Standardchemotherapie miteinander verglichen. Es zeigte sich, dass die Hochdosistherapie einen signifikanten Effekt auf das rezidivfreie Überleben hatte, jedoch - wenn überhaupt - nur einen sehr geringen Einfluss auf das brustkrebsspezifische bzw. das Gesamtüberleben. Darüber hinaus konnte keine Untergruppe definiert werden, die von einer Hochdosistherapie im besonderen Maße profitieren würde. Allerdings wurden zum Teil «alte» Chemotherapieschemata (z.B. ohne Taxane) eingesetzt. Auch der Benefit durch eine zusätzliche zielgerichtete Therapie, z.B. mit Trastuzumab, bleibt ungeklärt. Auf Grund dieser Daten gibt es derzeit weiterhin keine Indikation zur Hochdosistherapie in der adjuvanten Situation.

Gerber: Ich bin sicher, dass die Hochdosischemotherapie beim Mammakarzinom auch in Subkollektiven keine Renaissance erfahren wird. Es scheint eher so, dass dosisdichte Chemotherapieprotokolle - zumindest in Subgruppen - bedeutsamer werden. Mit der zunehmenden zielgerichteten Therapie wird es auch weniger um die «Menge» der eingesetzten Substanzen, als vielmehr um die Anwendung spezifisch wirkender Substanzen gehen.

Huober: In der metastasierten Situation sehe ich keine Renaissance der Hochdosistherapie, auch in der adjuvanten Situation sehe ich momentan dafür keine Hinweise; wenngleich es vielleicht ein selektiertes Patientinnenkollektiv geben könnte, welches von einer adjuvanten Hochdosischemotherapie profitieren könnte (z.B. Patientinnen mit $>10$ befallenen Lymphknoten). Da aber auch dosisdichte Therapien gerade bei den Hochrisikopatientinnen gute Ergebnisse gezeigt haben (z.B. ETC Studie) und zumindest bei HER2-positiven Patientinnen durch Trastuzumab eine Halbierung der Rezidivrate erreicht werden konnte, erscheint es sinnvoller, diese therapeutischen Strategien mit geringerer Toxizität weiter zu entwickeln.

Reitsamer: Eine Metaanalyse von 15 randomisierten Studien zeigte für die Hochdosischemotherapie im Vergleich zur Standardchemotherapie für das krankheitsfreie Überleben einen signifikanten Vorteil, jedoch für das brustkrebsspezifische und das Gesamtüberleben nur einen marginalen Vorteil. Im Zeitalter der Taxane und der zielgerichteten Therapien wird die Hochdosischemotherapie keinen Stellenwert mehr haben.

Schneewei $\beta$ : Dies ist trotz der Verbesserung des krankheits- freien Überlebens bei Hochrisikopatientinnen im Moment nicht abzusehen, da sich auch die konventionelle Systemtherapie ohne Stammzell-Unterstützung weiterentwickelt hat (neue Zytostatika wie die Taxane, zielgerichtete Therapien, dosisdichte Therapie). Die dosisdichte Therapie z.B. verbessert das Rezidiv-freie und das Gesamtüberleben bei Hochrisikopatientinnen (>10 Lymphknoten) bei wesentlich günstigerem Nebenwirkungsprofil. Außerhalb von kontrollierten Studien gibt es keine Indikation für eine Hochdosischemotherapie mit Stammzell-Unterstützung beim Mammakarzinom.

Thomssen: Für die adjuvante Hochdosischemotherapie konnte nur im Vergleich zu einer suboptimalen Vergleichstherapie ein klarer Vorteil für Patientinnen mit ausgedehntem Lymphknotenbefall gezeigt werden [2], im Vergleich zu einer optimal dosierten Standardtherapie dagegen nicht [3]. Eine Folgestudie ist geplant. Eine Empfehlung für den Einsatz in der Routine ist kurzfristig nicht zu erwarten. Für die metastasierte Situation waren die bisherigen Studienergebnisse enttäuschend, eine Renaissance ist hier auch mittelfristig sicher nicht zu erwarten.

\section{Frage 3: Gibt es einen neuen Standard zum Management von therapieassoziierten Knochendichte- minderungen bei Brustkrebspatientinnen? \\ Das Thema Knochenschutz unter Einnahme von Aroma- taseinhibitoren war eines der Schwerpunktthemen.}

Fehm: Bisher gab es zu diesem Thema nur die ASCO-Guidelines. Diese sehen eine Gabe von Bisphosphonaten bei Patientinnen mit einem T-Wert von $-2,5$ vor. Bei osteopenischen Patientinnen (T-Wert $-1,5$ bis $-2,5$ ) ist ein individualisiertes Vorgehen empfohlen. Da Frakturen z.B. vor allem bei osteopenischen Patientinnen unter Gabe von Aromataseinhibitoren (AI) auftraten, wurden im Rahmen des SABCS 2007 neue Guidelines für Patientinnen unter AI-Einnahme vorgestellt, die die Bisphosphonat-Gabe nicht nur vom T-Score, sondern auch vom Risikoprofil der Patientin abhängig machen. Des weiteren wurde die regelmäßige Knochendichtemessung empfohlen sowie die Gabe von Vitamin D und Calcium.

Gerber: Allen Patientinnen mit einem durch die Krebsbehandlung induzierten möglichen Knochenverlust (AI-Gabe) sollte zu allgemein präventiven Maßnahmen wie körperliche Aktivität, Kalzium- und Vitamin-D-Einnahme, normalem Körpergewicht, gesunder Ernährung und Änderungen im Lifestyle (Vermeidung von Kaffee, Cola, Nikotin usw.) geraten werden. Darüber hinaus sollte allen Patientinnen zur regelmäßigen Knochendichtebestimmung (DXA-Scan) geraten werden. Sollte eine Minderung der Knochendichte vorliegen bzw. sich diese unter der Therapie einstellen, so wäre hier aus meiner Sicht der Einsatz von Bisphosphonaten indiziert. Dazu gibt es jetzt ausreichend Daten. Beachtet werden sollte aber unbedingt der 
Zulassungsstatus der Bisphosphonate für die Therapie der Osteopenie/Osteoporose. Insofern würde ich nicht warten, bis die Osteoporose mit mehr als 2 Frakturen oder einem T-Wert im DXA-Scan unter -2,5 manifest wird, sondern rechtzeitig mit der Bisphosphonat-Therapie beginnen.

Huober: Gab es einen überhaupt einen alten Standard? Mit dem zunehmenden Einsatz der AI ist der Punkt Knochengesundheit vermehrt in den Vordergrund gerückt. Alle in San Antonio vorgestellten Studien, die sich mit dieser Fragestellung beschäftigt haben, zeigen, dass Bisphosphonate eine effektive Therapie sind, um einen therapiebedingten Knochendichteverlust aufzuhalten bzw. zu verbessern. Aber sollen wir allen immer alles geben? In der Zofast Studie, in der bei AITherapie eine sofortige Bisphosponattherapie mit einer verspäteten Bisphosphonattherapie (Indikation, wenn der TScore unter -2 fiel oder eine Fraktur auftrat, jeweils mit Zometa alle 6 Monate) verglichen wurde, zeigte sich nach 36 Monaten Follow-Up in der Sofortgruppe eine Zunahme der Knochendichte und im Vergleich dazu in der verspäteten Gruppe eine Abnahme der Knochendichte. Allerdings war die Frakturrate, also der klinisch relevante Faktor, ohne signifikante Unterschiede (wenngleich die Studie dafür leider nicht gepowert war).

Reitsamer: Die 3. Generation der Aromatasehemmer hat definitiv einen negativen Einfluss auf die Knochendichte. Alle osteopenischen Patientinnen unter AI sollen Bisphosphonate und Kalzium verabreicht bekommen und regelmäßig Osteodensitometriekontrollen erhalten. Denosumab, ein humaner monoklonaler Antikörper für RANKL (receptor activator of nuclear factor $\kappa \mathrm{B}$ ligand) zeigt bei osteopenischen Patientinnen eine Zunahme der Knochendichte (signifikanter Vorteil gegenüber Placebo), wird derzeit aber noch nur in Studien angewandt.

Schneewei $\beta$ : Kein neuer Standard, aber der bisherige Standard sollte konsequent befolgt, d.h. in Risikosituationen, wie z.B. bei Einnahme eines AI, die Knochendichte einmal pro Jahr kontrolliert werden. Tritt eine Osteoporose ein, sollte eine Therapie mit einem Bisphosphonat erfolgen. Die prophylaktische Gabe von Bisphosphonaten zur Verhinderung einer Osteoporose kann noch nicht allgemein empfohlen werden.

Thomssen: Zunächst muss das Ausmaß eines möglichen Knochendichteverlustes bewertet werden. Die regelmäßige Kontrolle der Knochendichte (z.B. mittels DXA) kann dafür sicher empfohlen werden. Bei Risikopatientinnen gilt der Einsatz von Bisphosphonaten als sinnvoll. Aus den Erfahrungen zur steroidinduzierten Osteoporose können weitere Empfehlungen abgeleitet werden: sportliche Belastung und Vermeidung eines zu niederigen Körpergewichtes $(\mathrm{BMI}<18)$. Auch die Substitution von Kalzium und Vitamin D ist sicher effektiv, wird aber durch die kürzlich publizierten Daten zum erhöhten kardiovaskulären Risiko in Frage gestellt [4].

\section{Frage 4: Welche Patientin muss ein Bisphosphonat erhalten?}

Fehm: Für Mammakarzinompatientinnen mit AI-Einnahme wurden entsprechende Guidelines vorgestellt. Diese sieht eine Bisphosphonat-Gabe vor bei i). Allen Patientinnen mit mindestens 2 Risikofaktoren (T-Score zwischen -1,5 und -2, Alter $>65$ Jahre, niedriger BMI $\left(<20 \mathrm{~kg} / \mathrm{m}^{2}\right)$, Hüftfrakturen in der Familie, eigene Fakturen, Rauchen, Kortisoneinnahme), ii) Patientinnen mit einem T-Score unter -2, iii) Patientinnen mit einem T-Score über -2 aber einem Abfall von $5 \%$ in der Knochendichte-Verlaufskontrolle.

Gerber: Siehe meine Antwort zu Frage 3. Sobald eine Minderung der Knochendichte vorliegt oder sich diese unter der Therapie einstellt, ist aus meiner Sicht der Einsatz von Bisphosphonaten indiziert.

Huober: In San Antonio wurde von P. Hadji ein Algorythmus zum praktischen Vorgehen bei AI-Therapie vorgestellt, der mir sehr praktikabel erscheint und der beschreibt, welche Patientin ein Bisphosphonat erhalten sollte und welche erst einmal nicht. Dies kann auch auf die prämenopausale Patientin übertragen werden. Sollten Bisphosphonate in den abgeschlossenen Studien auch als adjuvante Therapie einen Effekt zeigen, muss die Frage neu diskutiert werden.

Reitsamer: Alle osteopenischen Patientinnen unter AI sollen Bisphosphonate und Kalzium verabreicht bekommen und regelmäßig Osteodensitometriekontrollen erhalten.

Schneeweiß: Jede Patientin mit manifester Osteoporose, mit osteolytischen Knochenmetastasen, schmerzhaften osteoplastischen Knochenmetastasen oder einer tumorinduzierten Hyperkalzämie sollte Bisphosphonate erhalten.

Thomssen: Bisphosphonate müssen eingesetzt werden bei Hyperkalzämie, Knochenmetastasen (insbesondere bei osteolytischen Knochenmetastasen) und tumortherapieinduzierter Osteopenie oder Osteoporose. Bisphosphonate können auch vorteilhaft sein zur Verhinderung von Knochenmetastasen («adjuvanter Einsatz») und zur Verhinderung eines Knochenmasseverlustes bei Patientinnen mit erhöhtem Osteoporoserisiko.

\section{Frage 5: Muss der Einsatz von Psychopharmaka bei Brustkrebspatientinnen unter endokriner Therapie neu definiert werden?}

Fehm: Da eine Interaktion zwischen CYP2D6-Inhibitoren (z.B. Fluoxetin, Paroxetin) und Tamoxifen besteht und diese die Metabolisierung von Tamoxifen zu wirksamen Substanzen behindern könnte, sollte auf diese Präparate bei Mammakarzinompatientinnen unter Tamoxifentherapie verzichtet werden. 
Gerber: Die simultane Einnahme von Psychopharmaka und Antihormonpräparaten bei Brustkrebspatientinnen scheint mir ein typisches US-Phänomen zu sein. Aus meiner Sicht ergibt sich hier für Deutschland kein Diskussionsbedarf.

Huober: Zumindest bei Patientinnen mit Tamoxifentherapie muss die Psychopharmaka-Gabe überdacht werden, da bestimmte Antidepressiva (z.B. Paroxetin), die auch zur Behandlung klimakterischer Beschwerden eingesetzt werden, die Metabolisierung von Tamoxifen zur letztendlich wirksamen Substanz (Endoxifen, 4-OH Tamoxifen) behindern können. Aktuelle Daten zeigen, dass durch die Reduktion dieser Metabolisierung die Wirksamkeit von Tamoxifen vermindert sein kann.

Reitsamer: Eines der wenigen Medikamente zur Therapie von mit endokriner Therapie assoziierten klimakterischen Beschwerden scheint Venlafaxin zu sein.

Schneeweiß: Nein, die Daten zur Assoziation von bestimmtem Cytochrom-CYP2D6-Genotypen mit der Wirkung von Tamoxifen sind interessant aber retrospektiv, Hypothesen-generierend. Sie müssen daher vor dem klinischen Einsatz erst in prospektiven Studien bestätigt werden. Stehen neben Tamoxifen aber andere endokrine Therapieoptionen zur Verfügung, sollten diese vor dem Hintergrund einer Einnahme von Psychopharmaka diskutiert werden.

Thomssen: Bereits in den Konsensus-Empfehlungen von St. Gallen 2007 [5] wird auf Vorsicht im Umgang mit Antidepressiva vom SSRI-Typ bei gleichzeitiger TamoxifenEinnahme hingewiesen. Neuere Daten zeigen, dass die Umwandlung von Tamoxifen in seine aktiven Metaboliten unter dem Einfluss von SSRI empfindlich gestört werden kann.

Frage 6: Sind wir einem Standard der adjuvanten endokrinen Therapie von Hormonrezeptor-positiven Brustkrebspatientinnen in der Postmenopause näher gekommen? Welche Patientin soll eine Up-frontTherapie erhalten, welche Patientin soll mit Tamoxifen beginnen?

Fehm: Auf Grund der aktuellen Datenlage sollten alle postmenopausalen Frauen einen AI erhalten. Ob Up-front oder Switch ist noch nicht abschließend geklärt. Dies werden vor allem die Daten der BIG 1-98 Studie (Switch vs. up-front AI) zeigen. Auf Grund der jetzigen Datenlage ist bei Frauen mit hohem Rezidivrisiko (z. B. nodalpositiv, G3) der AI-Einsatz up-front zu bevorzugen. Für alle anderen Patientinnen ist die Switch-Therapie (Tam $\rightarrow$ AI) geeignet. 5 Jahre Tamoxifen sollten nur Patientinnen mit sehr geringen Rezidivrisiko erhalten oder solche, bei denen Kontraindikationen gegen AI vorliegen. Falls die Patientin bereits 5 Jahre Tamoxifen erhal- ten hat und ein erhöhtes Rezidivrisiko aufweist, sollte eine erweiterte adjuvante Therapie (EAT) mit einem AI in Erwägung gezogen werden. Auf Grund der Ergebnisse der MA-17Studie erscheint dieses Vorgehen auch dann sinnvoll, wenn das therapiefreie Intervall bis zu 36 Monate betragen hat.

Gerber: Hierzu hat sich in letzter Zeit klar abgezeichnet, dass postmenopausale Patientinnen mit einem mittleren und hohem Rezidivrisiko eine Up-front-Therapie erhalten sollen. Patientinnen mit einem sehr geringen Rezidivrisiko und/oder höherem Alter können auch weiterhin auf Tamoxifen eingestellt werden. Patientinnen, die bereits 2-3 Jahre Tamoxifen bekommen haben, müssen meines Erachtens auf einen AI umgestellt werden. Mehrere Studien haben übereinstimmend gezeigt, dass der «Switch» mit einem Überlebensvorteil - unabhängig von der individuellen Risikosituation - verbunden ist. Für eine «extended» Therapie (Aromatasehemmer nach 5 Jahren endokriner Therapie) kommen unter Abwägung von Nutzen und Risiken aus meiner Sicht nur wenige Patientinnen in Frage. Dazu gehören alle nodal-positiven Patientinnen. Die Dauer der endokrinen Therapie sollte mindestens 5 Jahre betragen und kann individuell bei guter Verträglichkeit und in Abhängigkeit von der Risikosituation verlängert werden. Dies muss aber nicht um jeden Preis erfolgen.

Huober: Alles ist möglich, die derzeitige Datenlage lässt keinen Schluss zu, ob eine Up-Front- oder sequentielle Therapie bevorzugt werden sollte. Erst die Ergebnisse der BIG Studie mit Auswertung der sequentiellen Arme im Vergleich zu den schon ausgewerteten Monotherapiearmen mit Letrozol oder Tamoxifen werden hierzu Hinweise geben. Hinweise, welche Patientinnen von einer AI-Therapie schon zu Beginn profitieren könnten gibt eine retrospektive Analyse der BIG Studie. Hier waren Risikofaktoren für ein frühes Rezidiv in den ersten 2 Jahren untersucht worden. Dabei zeigte sich dass Lymphknotenbefall, Tumor $>2 \mathrm{~cm}$, ein schlechtes Grading, eine HER2-Überexpression, nur einer der beiden Hormonrezeptoren positiv oder eine vaskuläre Invasion Risikofaktoren für ein frühes Rezidiv waren. Auch bei diesen Patientinnen war die Therapie mit Letrozol im Vergleich zu Tamoxifen effektiver.

Reitsamer: Die Datenlage für den Vorteil von AI der 3. Generation gegenüber Tamoxifen in Hinblick auf das krankheitsfreie und jetzt auch das Gesamtüberleben ist evident. Die ATAC-100-Daten haben gezeigt, dass sich mit zunehmender Nachbeobachtungszeit der Vorteil von Anastrozol noch vergrößert (Carry-over-Effekt). Somit sollte möglichst bei allen Patientinnen zunächst mit dem AI begonnen werden. Für Patientinnen, die aufgrund von ausgeprägter Osteoporose ein erhöhtes Frakturrisiko tragen, oder bereits osteoporotische Frakturen erlitten haben, wird Tamoxifen aber eine Up-frontOption bleiben. Patientinnen, die AI aufgrund der Nebenwirkungen nicht einnehmen können oder wollen, sind ebenso Kandidatinnen für Tamoxifen. Die definitiven Vergleichs- 
daten für AI «up-front», Tamoxifen up-front oder einen Wechsel nach 2 Jahren liegen noch nicht vor.

Schneeweiß: Es gibt keine durchgreifenden neuen Erkenntnisse. Die Ergebnisse der ATAC Studie mit einem Follow-Up von 68 Monaten wurden bei der aktuellen Analyse mit einem Follow-Up von 100 Monaten bestätigt. Anastrozol up-front verbessert im Vergleich zu Tamoxifen bei postmenopausalen Patientinnen mit HR-positivem Mammakarzinom signifikant das krankheitsfreie Überleben, die rezidivfreie und die fernmetastasenfreie Zeit sowie die Rate an kontralateralen Zweitkarzinomen, aber nicht das brustkrebsspezifische oder das Gesamtüberleben. Der Carry-over-Effekt von Anastrozol ist größer als der von Tamoxifen. Es traten keine Langzeitschäden auf.

Thomssen: Nein. Die derzeitig bekannten Daten lassen keinen Schluss zu, ob die Up-front- oder Sequenz-Therapie mit AI bevorzugt werden sollte. Die 100-Monats-Analyse der ATACStudie belegt überzeugend auch die langfristig zunehmende günstige Wirkung (Carry-over-Effekt) einer adjuvanten AITherapie, ein Überlebensvorteil lässt sich aber weiter nicht belegen. Damit bleibt weiter offen, welche Patientinnen am meisten von AI statt Tamoxifen profitieren, welche von Tamoxifen ähnlich gut profitieren würden und zu welchem Zeitpunkt ein AI am besten eingesetzt werden sollten.

\section{Frage 7: Welchen Stellenwert hat die Ernährung von Brustkrebspatientinnen als Teil der adjuvanten Therapie? Nur Lifestyle oder solide Prognosebeeinflussung?}

Fehm: Basierend auf den Daten der WHEL-Studie, die in San Antonio von Pierce et al. vorgestellt wurden, gibt es weiterhin keine klaren Hinweise, dass eine «Low-fat-Diät» mit einem hohen Anteil an Gemüse, Obst und Ballaststoffen die Prognose der Erkrankung günstig beeinflusst. Hingegen gilt der positive Effekt von Sport und Gewichtsnormalisierung als gesichert.

Gerber: Ich bin überzeugt, dass durch Ernährung und Lifestyle die Prognose nach Mammakarzinom beeinflusst werden kann. Durch Aromatasehemmer und teure Chemotherapie kann das Rezidivrisiko um etwa 22-25\% vermindert werden. R. Chlebowsky konnte in seiner Studie zeigen, dass eine fettreduzierte Diät zu einer Verbesserung des Gesamtüberlebens - unabhängig vom Hormonrezeptorstatus - führt. Auch körperliche Aktivität (etwa 3-5 h/Woche moderates Gehen) trägt zu einer Verbesserung des Gesamtüberlebens bei. Folglich muss allen Patientinnen nach Brustkrebs zu einer fettreduzierten Diät mit Erzielung eines normalen BMI und körperlicher Aktivität geraten werden. Bekanntermaßen wird dies von den häufig älteren und übergewichtigen Patientinnen leider nur unzureichend umgesetzt. Vitamine, Nah- rungsergänzungsmittel, Spurenelemente usw. können insbesondere bei einer laufenden Anti-Tumortherapie negative Effekte haben und es sollte deshalb eher davon abgeraten werden.

Huober: Mehrere Studien haben gezeigt, dass es eine Verbindung zwischen Lifestyle-Faktoren wie Diät, körperlichem Training und Adipositas und der Prognose einer Brustkrebserkrankung gibt. In der Womens Intervention Nutrition Study ergab sich z.B. durch die Intervention mit einer fettarmen Diät nach Mammakarzinomdiagnose, welche auch eine Gewichtsabnahme bedingte, eine Risikoreduktion für ein Rezidiv um 26\%. Die Patientinnen sollten von Ihren in die Primärtherapie involvierten Ärzten über diese Möglichkeiten, selber aktiv die Prognose positiv zu beeinflussen, beraten werden.

Reitsamer: Ein erhöhter BMI ist mit einem erhöhten Brustkrebsrisiko verbunden. In der Primärprävention spielt also die Ernährung eine wichtige Rolle zur Risikoreduktion. Auch der Zusammenhang von Insulinresistenz und Mammakarzinom wird zunehmend wichtiger. Bei bereits erkrankten Frauen mit erhöhtem BMI kann durch Reduktion der Fettaufnahme und Gewichtsabnahme das rezidivfreie Überleben verbessert und das Mortalitätsrisiko gesenkt werden. Auch wenn die Datenlage noch nicht sehr konsistent ist, ist eine Ernährungsberatung mit Empfehlung zu fettarmer und faserreicher Ernährung auch aus generellen medizinischen Gründen sinnvoll.

Schneeweiß: Zur Prognoseverbesserung führen Sport und Gewichtsnormalisierung. Bestimmte, definierte Diäten oder Nahrungsergänzungsmittel konnten keinen positiven Effekt zeigen.

Thomssen: Die Ernährung spielt eine Rolle für die Prognose. Patientinnen mit einem normalen Körpergewicht (BMI) haben die günstigsten Krankheitsverläufe nach Brustkrebserkrankung. Eine «Niedrigfett-Diät» mit einem Fettkalorienanteil von weniger als $20 \%$ der Gesamtkalorien soll das Rezidivrisiko insbesondere für östrogenrezeptornegative Tumoren senken. Diese Befunde müssen sicher noch bestätigt werden; vor allem dürfte eine Beratung in diese Richtung aufgrund der gewöhnungsbedürftigen Geschmacksneutralität einer solchen Diät auf berechtigte Skepsis bei unseren Patientinnen stoßen. Von einseitigen Diätplänen anderer Art («nur Gemüse, nur Körner») muss abgeraten werden. Ein günstiger Einfluss auf die Erkrankung ist nicht nachzuweisen.

\section{Frage 8: Welche Patientin soll vor der Operation eine MRT der Brust erhalten?}

Fehm: Derzeit gibt es keinen präoperativen Indikationskatalog. Jedoch empfiehlt sich bei folgenden Fragestellungen eine MRT: Bei CUP-Syndrom, zur Differentialdiagnose Narbe ver- 
sus Lokalrezidiv bei Mammakarzinompatientinnen insbesondere bei Zustand nach brusterhaltender Therapie, bei Frauen mit verdächtigen Befunden nach Protheseimplantation, bei Frauen mit neu diagnostiziertem Mammakarzinom und dichtem Brustdrüsengewebe (ACR 4) zum Ausschluss der Multizentrizität, bei Hoch-Risiko-Patientinnen (BRCA 1/2-Mutationsträgerinnen) mit dichtem Brustdrüsengewebe zur intensivierten Vorsorge ab dem 25. Lebensjahr und zur Verlaufsbeurteilung vor und nach neoadjuvanter Chemotherapie.

Gerber: Ich sehe derzeit keinen Grund, eine Mamma-MRT vor einer brusterhaltenden Operation durchführen zu lassen. Dazu fehlen zum einen in Deutschland qualitätsgesicherte und flächendeckende Strukturen. Zum anderen handelt es sich um ein invasives Verfahren und die Überlegenheit der Mamma-MRT hinsichtlich einer Verringerung der Lokalrezidive nach brusterhaltender Therapie ist (meines Wissens) nicht erbracht worden. Es ist vielmehr zu befürchten, dass durch zunehmende Anwendung der Mamma-MRT die Rate der Mastektomien und auch die Kosten erhöht werden.

Huober: Nicht jede Patientin! Die MRT ist eine sehr sensitive Untersuchungsmethode, hat aber den Nachteil einer mäßigen Spezifität. Mehrere Studien haben gezeigt, dass die Rate unnötiger Interventionen bei nicht selektioniertem präoperativem Einsatz der Mamma-MRT erhöht ist. Auch besteht die Gefahr, dass es vermehrt zu «unnötigen» Mastektomien kommt. Zur Frage der klinischen Relevanz einer präoperativen MRT (Verbesserung der Lokalrezidivrate und des Überlebens) gibt es bisher auch keine Daten. Ich würde daher nur in besonderen Situationen, wie von der Kommission Mamma formuliert (z.B. bei sehr dichter Mamma oder Verdacht auf Multizentrizität), eine Indikation für eine präoperative MRT sehen.

Reitsamer: Im Prinzip sollte mit wenigen Ausnahmen jede Patientin mit gesichertem Mammakarzinom eine MRT beider Brüste erhalten, einerseits zur exakten Beurteilung der Tumorausdehnung und zur Detektion von Multifokalität/-zentrizität mit der Folge der Reduktion von Sekundäreingriffen nach brusterhaltender Operation, andererseits zum sicheren Ausschluss von kontralateralen Tumorfoci. In jedem Fall sollten Patientinnen vor und nach primär systemischer Therapie eine MRT der Brust erhalten, um eine optimale Operationsplanung durchführen zu können. Inwieweit eine MRT auch bei Patientinnen mit DCIS eingesetzt werden wird, lässt sich noch nicht endgültig beurteilen.

Schneewei $\beta$ : Eine MRT sollte jede Patientin erhalten, bei der präoperativ anhand von Inspektion, Palpation, Mammasonographie und Mammographie die Ausdehnung des Mammakarzinoms nicht mit ausreichender Sicherheit bestimmt werden kann. Dies ist häufig schwierig beim lobulär-invasiven oder hereditären Mammakarzinom.
Thomssen: Die MRT-Diagnostik der Brust kann für Beratung und Operationsplanung hilfreich sein. Allerdings ist es unklar, ob die Rate radikalerer Operationsverfahren durch die präoperative MRT-Untersuchung nicht unnötig angehoben wird. Empfohlen wird eine präoperative MRT der Brust bei familiärem Mammakarzinom sowie bei histologisch gesichertem lobulären Karzinom.

\section{Frage 9: Hat sich das Management des axillären Stagings aufgrund der aktuellen Studienergebnisse verändert?}

Fehm: Derzeit noch nicht. Änderungen des Managements sind möglicherweise in Zukunft bei Patientinnen mit neoadjuvanter Chemotherapie zu erwarten. Basierend auf aktuellen Leitlinien ist eine Sentinel-Node (SN)-Biopsie bei Patientinnen mit neoadjuvanter Behandlung noch nicht empfohlen. Um den Stellenwert des SN in der neoadjuvanten Situation ausreichend bewerten zu können, läuft derzeit in Deutschland die prospektive Studie «SENTINA» (sentinel node excision after neoadjuvant therapy).

Gerber: Auch hier sehe ich derzeit keine Änderung. Der SN wird bei $\mathrm{cN} 0$ der Standard bleiben. Bei $\mathrm{cN}+$ wird weiterhin die axilläre Lymphonodektomie indiziert sein. Bei «sehr alten» Patientinnen mit einem kleinen Mammakarzinom kann bei klinisch unauffälliger Axilla auch gänzlich auf die Evaluierung des axillären Lymphknotenstatus verzichtet werden.

Huober: Die axilläre SN-Biopsie ist Behandlungsstandard für Patientinnen mit T1-2-Tumoren und klinisch negativer Axilla. Offen ist noch der Einsatz des SN bei T3-Tumoren und nach neoadjuvanter Therapie. Diese Frage wird gerade in der SENTINA-Studie (Leitung: Dres. Kühn und Bauerfeind) untersucht. Offen ist auch noch, bei welcher Patientin nach einem positiven SN eine komplette axilläre Dissektion indiziert ist. Nur zirka $40-50 \%$ der Patientinnen mit positivem SN haben dann in der folgenden axillären Dissktion weitere positive Lymphknoten. Auch nach San Antonio bleibt jedoch die axilläre Dissektion nach positivem SN bis zum Vorliegen weiterer Studienergebnisse das Standardvorgehen. Denn auch Untersuchungen der NSABP-B32-Studie konnten keine Faktoren, die einen weiteren Lymphknotenbefall sicher ausschließen, definieren.

Reitsamer: Die SN-Biopsie allein ist ein etabliertes Verfahren zum axillären Staging bei nodal negativen Patientinnen und primärer Operation. Einschränkungen gibt es nach wie vor bei Patientinnen nach primär systemischer Therapie, obwohl auch hier die Datenlage immer konklusiver wird. Die Frage, ob alle Patientinnen mit positivem SN eine Axilladissektion benötigen, ist nach wie vor nicht beantwortet. Es gibt verschiedene Ansätze, um bei positivem SN den Status der Non-Sentinel-
66

Breast Care 2008:3:61-67 
lymphknoten vorherzusagen. Nomogramme beziehungsweise multivariate logistische Regressionsanalysen mit mehr oder weniger Variablen (Anzahl der positiven SLNs, Anzahl der entfernten SLNs, Lymphovaskuläre Invasion, Größe der SNMetastase, klinische Tumorgröße...) haben eine relativ gute Wahrscheinlichkeit den Non-Sentinellymphknoten-Status vorauszusagen, können aber nicht generell empfohlen werden. Eine neue RT-PCR Methode (GeneSearch ${ }^{\mathrm{TM}}$ BLN assay) könnte möglicherweise die Anzahl an sekundären Axilladissektionen reduzieren durch die höhere Sensitivität in der Detektion von Mikro- oder Makrometastasen des SN verglichen mit der Gefrierschnittdiagnostik.

Schneeweiß: Im klinischen Alltag: nein. Ob nach einer positiven Sentinel-Lymphonodektomie in bestimmten Situationen (pT1, pN1a (1/>4sn), L0) auf eine komplettierende axilläre Lymphonodektomie verzichtet werden kann, bedarf der weiteren Untersuchung in klinischen Studien.

Thomssen: Das Management des axillären Stagings hat sich nicht wesentlich verändert. Bestätigt haben sich die Erfahrungen, dass das Sentinelverfahren auch nach vorausgegangener Chirurgie der Brust sensitiv ist. Das Sentinelverfahren sollte aber nicht dazu führen, die Indikation zur invasiven Axilladiagnostik weiter zu stellen. Die Sentinel-Exzision ist weiterhin nicht indiziert bei kleinen DCIS, sowie bei Mastektomie in prophylaktischer Intention.

\section{Literatur}

1 Harris L, Fritsche H, Mennel R, Norton L, Ravdin P, Taube S, Somerfield MR Hayes DF, Bast RC Jr; American Society of Clinical Oncology: American Society of Clinical Oncology 2007 update of recommendations for the use of tumor markers in breast cancer. J Clin Oncol 2007;25:5287-5312.

-2 Nitz UA, Mohrmann S, Fischer J, Lindemann W, Berdel WE, Jackisch C, Werner C, Ziske C, Kirchner H, Metzner B, Souchon R, Ruffert U, Schütt G, Pollmanns A, Schmoll HJ, Middecke C, Baltzer J, Schrader I, Wiebringhaus H, Ko Y, Rösel S, Schwenzer T, Wernet P, Hinke A, Bender HG, Frick M; West German Study Group: Comparison of rapidly cycled tandem high-dose chemotherapy plus peripheral-blood stem-cell support versus dose-dense conventional chemotherapy for adjuvant treatment of high-risk breast cancer: results of a multicentre phase III trial. Lancet 2005;366:1935-1944. Erratum in: Lancet 2006;367:730.

3 Bergh J, Wiklund T, Erikstein B, Lidbrink E, Lindman H, Malmström P, Kellokumpu-Lehtinen P, Bengtsson NO, Söderlund G, Anker G, Wist E, Ottosson S, Salminen E, Ljungman P, Holte H, Nilsson J, Blomqvist C, Wilking N: Tailored fluorouracil, epirubicin, and cyclophosphamide compared with marrow-supported high-dose chemotherapy as adjuvant treatment for high-risk breast cancer: a randomised trial. Scandinavian Breast Group 9401 study. Lancet 2000;356: 1384-1391. Erratum in: Lancet 2000;356:2196.

4 Bolland MJ, Barber PA, Doughty RN, Mason B, Horne A, Ames R, Gamble GD, Grey A, Reid IR: Vascular events in healthy older women receiving calcium supplementation: randomised controlled trial. BMJ 2008;336:262-266.

$\checkmark 5$ Goldhirsch A, Wood WC, Gelber RD, Coates AS, Thürlimann B, Senn HJ; 10th St. Gallen conference: Progress and promise: highlights of the international expert consensus on the primary therapy of early breast cancer 2007. Ann Oncol 2007;18:1133-1144. Erratum in: Ann Oncol 2007;18:1917.

\section{Teilnehmer}

Prof. Dr. med. Tanja Fehm

Universitätsfrauenklinik Tübingen

Calwerstr. 7, 72076 Tübingen, Germany

Tel. +4970712982257

E-Mail tanja.fehm@t-online.de

Prof. Dr. med. Bernd Gerber

Universitätsfrauenklinik und Poliklinik

am Klinikum Südstadt der Hansestadt Rostock

Südring 81,18059 Rostock, Germany

Tel. +49 381 4401-4500

E-Mail bernd.gerber@med.uni-rostock.de

Prof. Dr. med. Jens Huober

Senologiezentrum Ostschweiz SENZO

Kantonsspital St. Gallen

9007 St. Gallen, Switzerland

Tel. +41 71 494-1177, Fax -6368

E-Mail jens.huober@kssg.ch

PD Dr. med. Roland Reitsamer

Universitätsklinik für Spezielle Gynäkologie

Brustzentrum Salzburg

Müllner Hauptstraße 48, 5020 Salzburg, Austria

Tel. +43 662 4482-2571, Fax - 2572

E-mail r.reitsamer@salk.at

Prof. Dr. med. Andreas Schneeweiss

Universitäts-Frauenklinik

Voss-Strasse 9, 69115 Heidelberg, Germany

Tel. +49 6221 56-7856, Fax -7920

E-Mail andreas.schneeweiss@med.uni-heidelberg.de

Prof. Dr. med. Christoph Thomssen

Klinik und Poliklinik für Gynäkologie

Martin-Luther-Universität Halle-Wittenberg

Ernst-Grube-Strasse 40, 06097 Halle/Saale, Germany

Tel. +49 345 557-1847, Fax -1501

E-Mail christoph.thomssen@medizin.uni-halle.de 